\title{
V-Loc urethro-intestinal anastomosis during robotic cystectomy with orthotopic urinary diversion
}

\author{
Khanh N. Pham, MD; Bryan S. Sack, MD; R. Corey O'Connor, MD; Michael L. Guralnick, MD; \\ Peter Langenstroer, MD; William A. See, MD; Kenneth Jacobsohn, MD
}

Department of Urology, Medical College of Wisconsin, Milwaukee, WI

Cite as: Can Urol Assoc J 2013;7(11-12):e663-6. http://dx.doi.org/10.5489/cuai.508

Published online November 8, 2013.

\section{Abstract}

Introduction: Robotic-assisted radical cystectomy (RARC) is an emerging minimally invasive alternative for the treatment of invasive bladder cancer. The V-loc (Covidien, Mansfield, MA) suture is a unidirectional barbed suture that provides even distribution of tension. We determined the rate of urinary leak at the urethrointestinal anastomosis following orthotopic neobladder construction performed with V-loc suture.

Methods: We retrospectively reviewed charts on all patients that underwent RARC with orthotopic neobladder urinary diversion performed with a V-loc suture from February 2010 to February 2012. The urethro-intestinal anastomosis was evaluated for urinary leak by cystogram at 3 to 4 weeks, postoperatively.

Results: In total, 11 patients were available for analysis. The mean patient age was 57.2 years (range: $47-71$ ). The average clinical follow-up was 8 months (range: 4-15). On surgical pathology, 8 $(73 \%)$ patients had pT2 or less disease, 3 (27\%) had pT3/T4 disease, and 1 (9\%) had $\mathrm{N}+$ disease. The mean intraoperative blood loss was $315 \mathrm{~mL}$ (range: 150-600) and the average operative time was 496 minutes (range: 485-519). No patient $(0 \%)$ demonstrated a urinary leak at the urethro-intestinal anastomosis on postoperative cystogram. Eight patients (73\%) were continent by 4 months, postoperatively.

Conclusions: Orthotopic neobladder urethro-intestinal anastomosis can be performed effectively and safely with V-loc suture with an acceptably low urinary leak rate.

\section{Introduction}

Radical cystectomy remains the gold standard therapy for muscle invasive urothelial carcinoma of the bladder and is an effective oncologic treatment for patients with high-grade, recurrent, noninvasive urothelial bladder tumours. Roboticassisted radical cystectomy (RARC) has recently gained popularity as a minimally invasive alternative to traditional open radical cystectomy. To date, several single-centre retrospective case series and a large, multi-institutional analysis report acceptable oncologic and perioperative outcomes. ${ }^{1-5}$

The urethro-intestinal anastomosis of the orthotopic neobladder is difficult to perform during RARC due to length of the neobladder mesentery and patient positioning in steep Trendelenburg, which may hinder neobladder decent. Several techniques for suture placement have been described using both open and laparoscopic approaches. ${ }^{6-8}$ We have performed these anastomoses laparoscopically and have started using the V-loc (Covidien, Mansfield, MA) suture. The suture is a unidirectional barbed suture that grasps tissue at numerous points, providing even distribution of tension. In robotic-assisted radical prostatectomy (RARP), it was found to reduce set-up and anastomosis time, as well as cost. ${ }^{9}$ To date, there have been no studies to evaluate the efficacy of the V-loc suture when performing the urethrointestinal anastomosis following RARC with the orthotopic neobladder. We present our initial results using the $\mathrm{V}$-loc suture for construction of the urethro-intestinal anastomosis in orthotopic neobladders. Specifically, we determined the rate of urethro-intestinal anastomotic leak, rate of postoperative daytime continence, and short-term complications.

\section{Methods}

Following institutional review board approval, we retrospectively reviewed the medical records of all patients that underwent RARC with urinary diversion from February 2010 to February 2012 by a single, fellowship-trained urologic oncologic surgeon (KMJ). All urinary diversions were constructed by 1 of the 2 fellowship-trained urologic reconstructive surgeons (RCO or MLG). Exclusion criteria included patients with urinary diversion other than orthotopic ileal neobladder, urethrointestinal anastomosis performed other than with V-loc suture, absence of clinical follow-up, or previous pelvic radiation. 
We recorded preoperative characteristics, including patient age, gender, American Society of Anesthesiologists (ASA) score, whether or not patients received neoadjuvant chemotherapy and history of prior pelvic radiation. Intraoperative parameters, including operative time and surgical blood loss, were noted. Final pathology, including TNM stage and mean number of lymph nodes removed, were also noted.

The integrity of the urethro-intestinal anastomosis was evaluated for urinary leak at 3 to 4 weeks postoperatively with a cystogram. Daytime continence, defined as patients self-reporting the need for 1 safety pad or less per 24 hours, was also extracted from the medical record. Postoperative complications were documented.

\section{Brief description of the technique}

The extirpative portion of the procedure, including the radical cystectomy and an extended pelvic lymph node dissection, is performed robotically. Following undocking of the robot, a $4-\mathrm{cm}$ infraumbilical midline incision is made. The ileal neobladder is then constructed extracorporally. Bilateral 5-Fr ureteral stents are utilized to bridge the ureteroenteric anastomoses. The stents are brought out through the lumen of a 22-Fr Malecot catheter placed through the neobladder, which serves as a suprapubic catheter.

After construction, the ileal neobladder is placed back into the abdomen. The midline incision is closed, pneumoperitoneum is re-established and the robot is re-docked. The following is the technique used to perform the urethrointestinal anastomosis.

1. Place a single 2-0 Vicryl suture at the 6 o'clock position posteriorly reapproximating the ileal neobladder to the urethra.

2. Create a double-armed 3-0 V-loc suture by using 2 individual 3-0 V-loc sutures and passing each suture through the loop of the other suture.

3. Pass the V-loc suture behind the 2-0 Vicryl suture.

4. Place the left $\mathrm{V}$-loc suture needle at the 7 o'clock position of the neobladder.

5. Place the right $\mathrm{V}$-loc suture needle at the $5 \mathrm{o}^{\prime}$ clock position of the neobladder.

6. Complete the urethro-intestinal anastomosis by running both the left and right arm of the $\mathrm{V}$-loc suture individually and anteriorly over an 18-Fr urethral catheter.

7. Tie each end of the V-loc suture anteriorly at the 12 o'clock position.

8. Test the anastomosis for a leak by instilling $120 \mathrm{~mL}$ of normal saline through the urethral catheter.

All patients who underwent RARC with neobladder or Indiana pouch urinary diversion were evaluated postoperatively with a cystogram or pouchogram, respectively. Loopogram was not routinely performed in patients that underwent ileal conduit urinary diversion. Specifically for the neobladder patients, this is performed 3 to 4 weeks postoperatively to evaluate for a urinary leak from the ureterointestinal anastomoses, neobladder, or the urethro-intestinal anastomosis. If no urinary leak is demonstrated on cystogram, the urethral catheter is removed. The suprapubic catheter is capped and the patient begins Valsalva voiding per urethra. Once efficient urethral voiding is demonstrated, the suprapubic catheter is removed.

\section{Results}

In total, 62 patients underwent RARC with urinary diversion from February 2010 to February 2012. Eleven patients, all male, met the inclusion criteria and were available for analysis. Mean clinical follow-up was 8 (range: $4-15$ ) months. The average patient age was 57.2 (range: $47-71$ ) years. The mean ASA score was 2.6 (range: 2-3). All patients received neoadjuvant chemotherapy. The mean intraoperative surgical blood loss volume was 315 (range: 150-600) mL. On surgical pathology, $8(73 \%)$ patients had pT2 or less disease, $3(27 \%)$ had pT3/T4 disease, and 1 (9\%) had N+ disease. The mean number of lymph nodes removed was 26 (range: 16-39). Mean operative time was 496 (range: 485-519) minutes (Table 1).

No patient $(0 \%)$ demonstrated a urinary leak at the urethro-intestinal anastomosis on postoperative cystogram. Eight patients $(73 \%)$ reported daytime continence by 4 months, postoperatively (Table 2 ). There were no early complications related to the urinary diversion or urethro-intestinal anastomosis.

\section{Discussion}

RARC is becoming the minimally invasive gold standard for the surgical management of bladder cancer at many tertiary medical centres. For those patients that qualify for orthotopic neobladder urinary diversion, many urologists are re-docking the robot after creating the neobladder extracorporeally prior to the urethro-intestinal anastomosis. This study investigates the efficacy and safety using the V-loc suture to perform a robotic-assisted urethro-intestinal anastomosis.

The urethro-intestinal anastomosis is technically challenging secondary to the short neobladder mesentery and the steep Trendelenberg positioning. There are different methods of performing the urethro-intestinal anastomosis. ${ }^{6-8}$ Patel describes pre-placing anastomotic sutures in the posterior lip of the neobladder, closing the fascia, re-establishing pneumoperitoneum, and then finishing the anastomosis in laparoscopic fashion using running continuous suture. ${ }^{6}$ Alternatively, Pruthi and colleagues completed the anastomosis in an open fashion with interrupted sutures. ${ }^{7}$ Prior to the use of the V-loc suture at our institution, we performed 


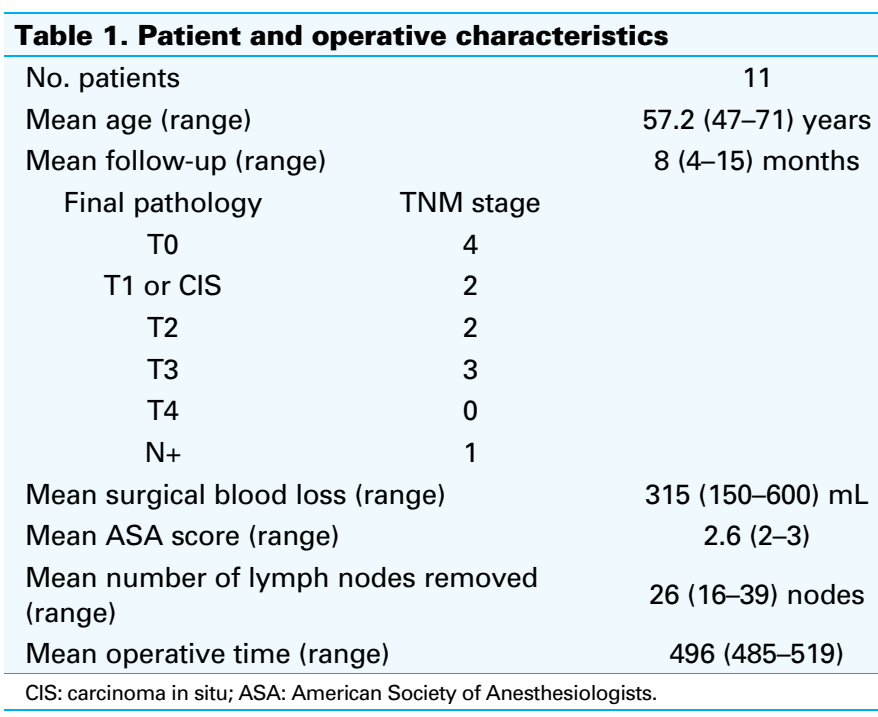

the former laparoscopic method with continuous running absorbable sutures. Our utilization of the V-loc suture for the vesico-urethral anastomosis for RARP cases led to the investigation of its use for the orthotopic neobladder urethrointestinal anastomosis.

The V-loc suture has been described by Zorn and colleagues to aid in performing the vesico-urethral anastomosis during RARP. This single-surgeon prospective trial demonstrated a reduction in nurse set-up time (31 vs. 294 seconds), reconstruction time (13.1 vs. 20.8 minutes), cost (48.05 vs. $70.25 \$ C A N)$ and need to readjust suture tension or placement of additional suture clips (6\% vs. $24 \%$ ) using V-loc suture compared to standard monofilament suture. No delayed anastomotic leaks or bladder neck contractures were observed in either group and pad-free continence outcomes were similar between the 2 groups at $1-, 3-$, and 6-month intervals. ${ }^{9}$ Sammon and colleagues have also published similar findings on use of the V-loc suture compared to standard monofilament suture for the vesico-urethral anastomosis in RARP. This multi-surgeon prospective trial showed a reduction in posterior reconstruction time (3.3 vs. 4.3 minutes) and vesico-urethral anastomosis time (10.1 vs. 13.8 minutes). Perioperative and postoperative urinary functional outcomes were similar between both groups. ${ }^{10}$

Extrapolating upon the experience of Zorn and Sammon, as well as our own with use of V-loc suture for RARP, we investigated its applicability for performing the urethrointestinal anastomosis in orthotopic neobladder construction during RARC. Haber and colleagues published findings of a $0 \%$ urinary leak rate (0/19 patients) when performing openassisted laparoscopic orthotopic neobladder construction. ${ }^{11}$ Our $0 \%$ urinary leak rate using the $\mathrm{V}$-loc suture is equal to the reported urinary leak rate in the literature when performing the urethro-intestinal anastomosis with interrupted sutures. The daytime continence rate at 4 months postopera-

\section{Table 2. Anastomotic leak and continence results}

$\begin{array}{lc}\text { Urethro-intestinal anastomotic urinary leak } & 0 \%(0 / 11) \\ \text { Daytime continence } & 73 \%(8 / 11)\end{array}$

tively of $73 \%$ in this case series is somewhat lower than the rate of $92 \%$ at 12 months postoperatively described in the literature for open series. ${ }^{8}$ However, Steers and colleagues found that patients with orthotopic diversion continued to have improvement over the first 6 to 12 months, and potentially daytime continence of our patient cohort will continue to improve over time. ${ }^{12}$ Certainly, as our patient cohort increases, we will be better able to understand the efficacy of the V-loc suture on perioperative and postoperative outcomes.

There are limitations in our study. It was retrospective in design with a small number of patients and short follow-up. Furthermore, we did not have a formal control group to provide specific comparisons with regards to operative time and superiority in urinary leak rate compared to performing the urethro-intestinal anastomosis with either interrupted sutures or a running monofilament suture. As well, our continence data are based on patient-reported pad usage, which does not necessarily accurately reflect the magnitude of incontinence. Furthermore, we used a definition of continence that includes up to 1 pad per 24 hours (i.e., social continence) and thus does not imply complete dryness. However, this is a definition that is often used for postoperative continence. ${ }^{13}$ Nonetheless, we have demonstrated that the V-loc suture can be safely and easily used to perform the urethrointestinal anastomosis in orthotopic neobladder construction during RARC with excellent short-term outcomes.

\section{Conclusions}

The orthotopic neobladder urethro-intestinal anastomosis can be robotically performed effectively and safely with the V-loc suture. The urinary leak rate is acceptably low and comparable to the reported rate in the literature.

Competing interests: Dr. Pham, Dr. Sack, Dr. Langenstroer, Dr. See, and Dr. Jacobsohn all declare no competing financial or personal interests. Dr. O'Connor has received honoraria from Astellas. Dr. Guralnick is a member of the Speakers Bureau for Astellas and Allergan. He is also a principal investigator for InControl Medical.

This paper has been peer-reviewed.

\section{References}

1. Dasgupta P, Rimington P, Murphy D, et al. Robotic assisted radical cystectomy: Short to medium-term oncologic and functional outcomes. Int I Clin Pract 2008;62:1709-14. http://dx.doi.org/10.1111/ j.1742-1241.2008.01858.x 
Pham et al.

2. Wang GJ, Barocas DA, Raman JD, et al. Robotic vs open radical cystectomy: Prospective comparison of perioperative outcomes and pathological measures of early oncological efficacy. BJU Int 2008;101:89-93 http://www.ncbi.nlm.nih.gov/pubmed/17888044

3. Smith Ab, Raynor, Amling CL, et al. Multi-institutional analysis of robotic radical cystectomy for bladder cancer: perioperative outcomes and complications in 227 patients. J Laparoendosc Adv Surg Tech A 2012;22:17-21. http://dx.doi.org/10.1089/lap.2011.0326

4. Pruthi RS, Nielsen ME, Nix J, et al. Robotic radical cystectomy for bladder cancer: surgical and pathological outcomes in 100 consecutive cases. J Urol 2010;183:510-4. http://dx.doi.org/10.1016/i. juro.2009.10.027

5. Pruthi RS, Wallen EM. Robotic assisted laparoscopic radical cystoprostatectomy: operative and pathological outcomes. J Urol 2007;178(3 Pt 1):814-8. http://dx.doi.org/10.1016/i.juro.2007.05.040

6. Patel V. Robotic Urologic Surgery. 2nd ed. New York: Springer; 2012. http://dx.doi.org/10.1007/9781-84882-800-1

7. Pruthi RS, Wallen EM. Robotic-assisted laparoscopic radical cystoprostatectomy. J Eur Urol 2008;53:31022. http://dx.doi.org/10.1016/i.eururo.2007.03.067

8. Studer UE, Burckard FC, Schumacher $M$, et al. Twenty years experience with an ileal orthotopic low pressure bladder substitute—lessons to be learned. J Urol 2006;176:161-6. http://dx.doi.org/10.1016/ S0022-5347(06)00573-8
9. Zorn KC, Trinh QD, Jeldres $C$, et al. Prospective randomized trial of barbed polyglyconate suture to facilitate vesico-urethral anastomosis during robot-assisted radical prostatectomy: time reduction and cost benefit. BJU Int 2012;109:1526-32. http://dx.doi.org/10.1111/i.1464-410X.2011.10763.x. Epub 2012 Jan 5.

10. Sammon J, Kim TK, Trinh QD, et al. Anastomosis during robot-assisted radical prostatectomy: randomized controlled trial comparing barbed and standard monofilament suture. J Urol 201 1;78:572-9. http:// dx.doi.org/10.1016/i.urology.2011.03.069

11. Haber GP, Campbell SC, Colombo JR Jr, et al. Perioperative outcomes with laparoscopic radical cystectomy: "pure laparoscopic" and "open-assisted laparoscopic" approaches. J Urol 2007;70:910-5. http://dx.doi. org/10.1016/i.urology.2007.07.012

12. Steers WD. Voiding dysfunction in the orthotopic neobladder. World J Urol 2000;18:330-7. http:// dx.doi.org/10.1007/s003450000146

13. Castle $\mathrm{EP}$, Andrews $\mathrm{PE}$, Itano $\mathrm{N}$, et al. The male sling for post-prostatectomy incontinence: mean followup of 18 months. J Urol 2005;173:1657-60. http://dx.doi.org/10.1097/01.ju.0000154782.86431.41

Correspondence: Dr. Bryan S. Sack, Medical College of Wisconsin, Department of Urology, 9200 West Wisconsin Ave., Milwaukee, WI 53226; bsack@mcw.edu 